\title{
(Non-)variabilité du rythme en français canadien
}

\author{
Svetlana, Kaminskaïa \\ Université de Waterloo, 200, University Ave. W., Waterloo (Ontario), Canada, N2L 3G1 \\ skaminsk@uwaterloo.ca
}

\begin{abstract}
Résumé. Le français ontarien partage la grammaire phonologique avec son parent québécois, tout en présentant des divergences conditionnées par la distance géographique et par le contact avec l'anglais. Le rythme du français ontarien a fait objet d'une série d'études récentes utilisant des mesures rythmiques, mais cet aspect prosodique n'a pas démontré d'influence du contexte minoritaire et du contact avec l'anglais. De plus, les données du contexte minoritaire ont montré une rythmicité plus syllabique (française) que celles des contextes majoritaires. Les mesures rythmiques seules manquant de capacité d'explication, pour explorer plus précisément les origines de ces résultats inattendus, nous comparons les données du français ontarien en contact et du français québécois, et explorons la typologie syllabique, la longueur et la durée des groupes accentuels, les rapports de durée des syllabes au sein d'un groupe accentuel et l'intensité des voyelles dans les limites de ce groupe. Des enregistrements spontanés ont servi à cette étude. Les résultats démontrent des similarités mais aussi des différences importantes qui tiennent à la distribution des durées syllabiques et à l'intensité des voyelles, et suggèrent qu'en français, en contexte minoritaire, l'intensité serait un corrélat acoustique de l'accent rythmique primaire et secondaire.
\end{abstract}

\begin{abstract}
Rhythmic (in)variability in Canadian French. While sharing phonology with the parent Quebec variety, French spoken in Ontario shows differences that are due to the contact with English and to the geographical distance from Quebec. However, a number of recent studies focusing on prosodic rhythm and applying rhythm metrics did not reveal any effect of language contact. Moreover, not only they did not observe in Ontario French a more stress-timed pattern proper to the majority language, they found that the minority French data has an even more syllable-timed (French) pattern. Rhythm metrics alone are not able to explain these unexpected results, therefore, in order to better understand them, we are undertaking a comparative analysis of Ontario and Quebec spontaneous data focusing on syllabic typology, length and duration of the stress group, syllable duration ratios within it, and vowel intensity. Results reveal rhythmic similarities but also important differences that pertain to the duration ratios and to the distribution of the intensity within the stress group, suggesting that the intensity is an important acoustic correlate of primary and secondary stress in French spoken in contact with English.
\end{abstract}

\section{1 Études antérieures et questions de recherche}

Il y a deux grands dialectes du français au Canada - le français acadien, parlé dans les provinces maritimes, et le français québécois, parlé au Québec et à l'ouest de la province dans le reste du pays. Au cours des dernières décennies, plusieurs études ont démontré une riche variabilité linguistique à l'intérieur de chaque dialecte. Au niveau prosodique, pendant une longue période, les variétés du français québécois n'ont pas été distinguées du dialecte d'origine et ont été décrites comme similaires, voire identiques. La tendance récente, cependant, cherche à nuancer la variation prosodique dans les variétés du français parlées à travers le Canada, et des analyses de la prosodie des variétés acadiennes (Cichocki 1997; Cichocki et al. 2014), québécoise (Kaminskaïa 2005, 2015), albertaine (Mzemba 2016) et ontariennes (Kaminskaïa 2013; 2014 ; Kaminskaïa et al. 2015; Tennant 2011) ont été proposées.

Ainsi, le français ontarien et le français québécois partagent la grammaire intonative et présentent un inventaire tonal similaire (Kaminskaïa 2013). Cependant, les particularités intonatives suivantes distinguent le français ontarien parlé en contexte minoritaire des variétés majoritaires : prédominance des contours descendants de continuité (ibid.), présence de la ligne de régression (Cichocki et Lepetit 1986) et des contours upstep (Kaminskaïa et Poiré 2012), et alignement plus tardif des pics mélodiques (Kaminskaïa 2012).

En ce qui concerne le rythme, selon les analyses antérieures, le français québécois montre des syllabes accentuées plus courtes et des syllabes inaccentuées plus longues qu'en français standard (Boudreault 1968). Le français ontarien, quant à lui, présente une syllabe pénultième plus saillante que le français standard (Vinay 1955) et un rythme irrégulier (Robinson 1968), mais le pattern accentuel et la nature de l'accent restent français - accent final de durée (Williams et Poiré 2007). Nous pouvons observer cela dans le Tableau 1, qui compare les durées syllabiques par rapport à la durée de la syllabe finale dans un groupe accentuel de quatre syllabes en français standard, québécois et ontarien. Dans ce tableau, les données pour le français ontarien en contextes majoritaire et minoritaire sont présentées séparément, avec deux résultats pour le français ontarien parlé en contexte minoritaire - une étude moins récente de Robinson (1968) et plus récente de Kaminskaïa et al. (2009). 
Tableau 1. Proportion des durées moyennes (\%) des syllabes faisant partie des groupes rythmiques de quatre syllabes par rapport à la syllabe finale.

\begin{tabular}{|l|c|c|c|c|}
\hline & $\mathbf{4}^{\mathrm{e}}$ de la fin & $\mathbf{3}^{\mathrm{e}}$ de la fin & Pénultième & Syllabe finale \\
\hline $\begin{array}{l}\text { Français standard } \\
\text { (Robinson 1968) }\end{array}$ & $31,9 \%$ & $42,2 \%$ & $45,7 \%$ & $100 \%$ \\
\hline $\begin{array}{l}\text { Québec (Kaminskaïa et al. } \\
2009 \text { ) }\end{array}$ & $48,0 \%$ & $57,0 \%$ & $67,0 \%$ & $100 \%$ \\
\hline $\begin{array}{l}\text { Ontario, contexte } \\
\text { majoritaire (ibid.) }\end{array}$ & $62,0 \%$ & $59,0 \%$ & $62,0 \%$ & $100 \%$ \\
\hline $\begin{array}{l}\text { Ontario, contexte } \\
\text { minoritaire (ibid.) }\end{array}$ & $59,0 \%$ & $69,0 \%$ & $89,0 \%$ & $100 \%$ \\
\hline $\begin{array}{l}\text { Ontario, contexte } \\
\text { minoritaire (Robinson } \\
1968)\end{array}$ & $62,6 \%$ & $73,4 \%$ & $73,9 \%$ & $100 \%$ \\
\hline
\end{tabular}

Dans le Tableau 1, on remarque, en effet, que la proportion des syllabes inaccentuées en français standard est inférieure aux proportions des variétés canadiennes : ici elle varie de $31,9 \%$ à $45,7 \%$, alors que les valeurs dans les variétés canadiennes fluctuent entre $48,0 \%$ et $67,0 \%$ (Québec), entre 59,0\% et 62,0\% (Ontario, contexte majoritaire) et entre $59,0 \%$ et $89,0 \%$ (Ontario, contexte minoritaire). La syllabe initiale du groupe est la plus longue en français ontarien minoritaire $(62,6 \%)$ selon les données de Robinson (1968), en comparaison avec le français québécois $(48,0 \%)$, et surtout avec le français standard (31,9\%). La durée de cette syllabe, cependant, se rapproche de celles en français ontarien en contextes majoritaire (62\%) et minoritaire (59\%, Kaminskaïa et al. 2009). Les ressemblances des données ontariennes en contexte minoritaire avec les autres variétés se terminent ici, car elles montrent des durées syllabiques considérablement plus longues pour les syllabes pénultièmes $(73,9 \%$ et $89 \%)$ et antépénultièmes $(73,4 \%$ et $69 \%$ ) dans les études de Robinson (1968) et de Kaminskaïa et al. (2009). Il faut noter les différences importantes entre les valeurs ontariennes en contexte minoritaire rapportées en 1968 comparativement à celles rapportées en 2009 : les durées des syllabes initiales et antépénultièmes se sont réduites d'environ $4 \%$, alors que la durée de la syllabe pénultième a augmenté d'environ $15 \%$. Comme il s'agit d'études basées sur des corpus différents et sur un nombre réduit de participants (souvent, il s'agit des données venant d'une seule personne), il n'est pas possible d'affirmer l'importance de ces dissimilitudes.

À la différence de la variabilité ressortant du Tableau 1, les études antérieures sur l'isochronie (ou rythme prosodique) en français ontarien en contextes minoritaire et majoritaire (Kaminskaïa 2014; Kaminskaïa et al. 2015) et en français ontarien et québécois (Kaminskaïa 2015) constatent une rythmicité syllabique à travers l'ensemble des corpus. Ces analyses, qui s'appuient sur les mesures rythmiques ${ }^{2}$, non seulement réfutent l'hypothèse sur la convergence au rythme anglais en milieu minoritaire, mais suggèrent une rythmicité plus syllabique que dans les variétés majoritaires du français. Ainsi, la valeur plus basse de VarcoV et la valeur plus haute de $\% \mathrm{~V}$ en contexte minoritaire suggèrent un rythme plus syllabique dans cette variété par rapport au contexte majoritaire (Tableau 2). En même temps, les valeurs plus basses des $\Delta \mathrm{V}, \Delta \mathrm{C}$ et VarcoC en milieu minoritaire suggèrent une moindre complexité des structures syllabiques dans ce sous-corpus par comparaison avec le sous-corpus du contexte majoritaire, ce qui va de pair avec le rythme syllabique.

Tableau 2. Mesures rythmiques et débit en français ontarien des milieux minoritaire et majoritaire (adapté de Kaminskaïa et al. 2015). Les mesures rythmiques surlignées ont démontré des différences significatives.

\begin{tabular}{|l|c|c|c|c|c|c|c|}
\hline & $\mathbf{n P V I}-\mathbf{V}$ & $\mathbf{\Delta V}$ & $\boldsymbol{\Delta} \mathbf{C}$ & $\mathbf{\% V}$ & VarcoV & VarcoC & Débit (syll./s) \\
\hline Contexte majoritaire & 41,53 & 0,076 & 0,073 & 50,99 & 65,78 & 64,43 & 5,06 \\
\hline Contexte minoritaire & 42,3 & 0,055 & 0,047 & 54,35 & 49,39 & 52 & 5,1 \\
\hline
\end{tabular}

L'application du modèle Control and Compensation (voir note 2) dans Kaminskaïa (2014), cependant, a permis de caractériser la variété ontarienne en contexte minoritaire comme présentant un rythme intermédiaire-accentuel en fonction de l'âge des locuteurs - les participants jeunes ont présenté un rythme accentuel. Cela a réconcilié les tendances contradictoires suggérées par les mesures $\% \mathrm{~V}, \mathrm{VarcoV}, \Delta \mathrm{V}, \Delta \mathrm{C}$ et VarcoC, d'un côté, et les mesures nPVI$\mathrm{V}$, de l'autre côté. Finalement, en ce qui concerne la comparaison des données québécoises et ontariennes du contexte minoritaire, l'étude de Kaminskaïa (2015) a identifié le débit comme la seule différence significative entre les souscorpus : les locuteurs du français en contact ont parlé plus lentement que ceux du Québec. Les deux variétés ont même montré une rythmicité plus syllabique que dans les analyses antérieures du français européen. La combinaison du débit lent et d'une rythmicité régulière rapproche le français ontarien des autres variétés du français européen régional, et le distingue des variétés du français en contact qui se caractérisent par un rythme lent et une rythmicité irrégulière. Le français québécois, avec un débit rapide et un rythme régulier, se distingue du français standard qui a un débit rapide et un rythme relativement régulier (Kaminskaïa 2015). Ce résultat va de pair avec les autres études qui ont démontré une contribution du débit à la différenciation rythmique (Obin et al. 2012; Schwab et Racine 2013).

Le rythme très syllabique observé en français ontarien et québécois pourrait s'expliquer par une différence plus petite entre les proportions des durées des syllabes (voire des voyelles ${ }^{3}$ ) inaccentuées et accentuées, en comparaison du français standard où cette différence est plus grande (Tableau 1). Toutefois, la similarité des valeurs des mesures 
rythmiques dans les variétés québécoise et ontarienne en contact devrait aller de pair avec la similarité des proportions des durées syllabiques, ce qui n'est pas le cas (Tableau 1). Pour mieux comprendre ces résultats, nous proposons ici d'examiner les rapports duratifs dans ces deux variétés canadiennes sur un corpus plus grand.

Ensemble avec les rapports des durées des syllabes inaccentuées par rapport à la syllabe finale, nous allons comparer les proportions des durées des syllabes à l'intérieur d'un groupe accentuel pour nous concentrer sur la durée de la syllabe finale. Si la durée de la syllabe accentuée est réduite, nous pouvons supposer un changement des rôles des corrélats acoustiques dans l'accentuation, c'est-à-dire un changement de la nature de l'accent. Autrement dit, les différences entre les variétés (et l'effet de l'anglais) peuvent se manifester non pas au niveau de la rythmicité, mais au niveau des corrélats acoustiques de l'accent. Selon Lieberman (1960), la mélodie et l'intensité déterminent la nature de l'accent en anglais nord-américain. En français européen, ce sont la durée et la fréquence fondamentale qui le font (Cumming 2011). Alors, il est naturel de se demander si le rôle de l'intensité dans l'accentuation en français ontarien devient plus grand qu'en français québécois.

En effet, les rapports d'intensité, tout comme les changements des rapports duratifs, peuvent intervenir dans le pattern rythmique. Cela a été démontré par Cichocki et al. (2014) dans les variétés du français acadien parlé en contact avec l'anglais : dans cette étude, les mesures rythmiques basées sur les valeurs d'intensité ont montré plus de pouvoir distinctif entre les variétés acadiennes que les mesures basées sur les valeurs de durée. Ce résultat fait dire aux auteurs que l'intensité pourrait être un des corrélats acoustiques de l'accent en français acadien.

Le Tableau 1 suggère que le français ontarien en contexte minoritaire présente une prosodie particulière. Cependant, les résultats des analyses antérieures portant sur le rythme prosodique le qualifient de " très français » et ne soutiennent pas l'hypothèse de convergence à l'anglais. En même temps, d'autres analyses permettent de supposer que l'effet du contact linguistique pourrait se manifester par la nature de l'accent. Pour mieux comprendre la nature de la (non-)variabilité rythmique observée dans les études précédentes portant sur le français québécois et sur le français ontarien en milieu minoritaire, nous entreprenons cette analyse et examinons les aspects suivants : la typologie syllabique, la longueur et la durée des groupes accentuels, et les rapports des durées syllabiques à l'intérieur d'un groupe accentuel ${ }^{4}$. Pour explorer le rôle de l'intensité dans les patterns rythmiques, nous examinons également les moyennes de l'intensité normalisée des voyelles de ces groupes.

\section{Méthodologie}

Pour répondre aux questions posées et explorer les aspects rythmiques spécifiés, nous utilisons un sous-ensemble du corpus examiné dans l'étude du rythme prosodique par Kaminskaïa (2015) : les entretiens spontanés de six locuteurs des corpus Québec (FQ) et Windsor (FW, Poiré et Kelly 2003). Ces enregistrements font partie de la base de données du projet international Phonologie du français contemporain (PFC, Durand et al. 2009). Chaque échantillon est composé de trois femmes et trois hommes; l'âge des participants varie de 17 à 74 ans. À cette étape, nous nous concentrons sur les différences régionales; l'effet des variables d'âge et de sexe pourra être testé sur un corpus plus large.

Le traitement des enregistrements a été effectué dans Praat (Boersma et Weenink 2016); les pauses, les troncations, les hésitations, les alternances codiques, etc., ont été exclues de l'analyse. Le découpage initial semi-automatique en syllabes et en segments a été fait à l'aide de EasyAlign (Goldman 2011). La vérification manuelle, basée sur les contrôles auditif et visuel, a suivi l'étape du découpage; la resyllabation a été appliquée lorsque les cas de liaison et d'enchaînement la commandaient. La hiérarchie de sonorité et les préférences de la langue française ont aussi été prises en considération. Par exemple, nous avons divisé les groupes consonantiques commençant par un [s] de façon à garder cette consonne dans la coda de la syllabe précédente : «restaurant» a été syllabifié [rEs-to-rA)]; «absolument », [ap$\mathrm{s} \square$-ly-mA)], en dépit de [a-ps $\square$-ly-mA)], division requise par la hiérarchie de sonorité.

La structure syllabique a été établie à partir des frontières syllabiques, et la distribution des structures syllabiques nous a permis de juger de la typologie syllabique. À partir du nombre de syllabes et de la durée du signal analysés, nous avons calculé la vitesse d'articulation (ou le débit), en syllabes par seconde (syll./s). Le découpage des données en groupes rythmiques/accentuels, ou Syntagmes Accentuels (SAs), a suivi les contraintes spécifiées par Jun et Fougeron (2002) et adaptées à la parole spontanée par Kaminskaïa (2005). Le nombre de syllabes réalisées dans un SA a déterminé sa longueur, et sa durée a été mesurée en secondes. Les proportions de durée des syllabes par rapport à la durée du SA et les proportions des durées des syllabes inaccentuées par rapport aux syllabes accentuées ont été calculées pour les groupes accentuels de quatre syllabes pour comparer nos résultats avec les analyses antérieures dans le Tableau 1.

La normalisation de l'intensité a été calculée à l'aide de la cote Z. Pour cela, nous avons extrait de Praat les valeurs décibels $(\mathrm{dB})$ pour chaque SA de quatre syllabes. Ensuite, pour chaque SA, nous avons calculé l'intensité moyenne et l'écart-type et divisé la différence entre chaque valeur dB et la moyenne par la valeur de l'écart-type: $(N-$ moyenne)/écart-type. Par la suite, pour chacune des quatre voyelles du SA, nous avons calculé les moyennes des valeurs d'intensité normalisées.

Les différences entre les sous-corpus ont été testées à l'aide des tests $t$ bilatéraux à deux échantillons. Pour l'analyse des différences des valeurs d'intensité observées dans les groupes accentuels contenant un seul accent final ou bien un accent final et un accent initial (voir plus bas), nous avons appliqué les tests ANOVA 2x2 (variété - FW c. FQ; nombre d'accents - un vs deux). 


\section{Résultats}

\subsection{Portrait général}

Nous présentons d'abord le portrait général de chaque sous-corpus pour passer ensuite à la typologie syllabique et à la distribution de durée syllabique et d'intensité vocalique à travers un SA.

Dans le sous-corpus ontarien, la durée totale des enregistrements analysés est de 1405,6 s, mais après l'exclusion des pauses, des hésitations, des alternances de codes, etc., la durée utilisée a totalisé 1095,22 s, ou 91,27 s par personne en moyenne (Tableau 3). Cela a donné 358,5 syllabes par personne, avec un minimum de 123 syllabes chez Patrice et $^{5}$ un maximum de 632 syllabes chez Rémie. Ce matériel a fourni entre 35 SAs (Patrice) et 195 SAs (Rémie) par locuteur, soit 115,67 SAs en moyenne. La longueur des groupes accentuels a varié de 2,47 syll. (Raymond) à 3,59 syll. (Éliane), avec une moyenne de 3,22 syll. En ce qui concerne la durée moyenne des SAs, elle se situe à 0,69 $\mathrm{s}$ et varie de $0,51 \mathrm{~s}$ (Rémie) à $0,79 \mathrm{~s}$ (Patrice). Le débit d'articulation moyen des participants du corpus Windsor est de 4,64 syll./s; Raymond a parlé le plus lentement (3,96 syll./s), alors que Rémie a articulé le plus vite (5,19 syll./s).

Tableau 3. Découpage, longueur et durée des SAs : corpus FW.

\begin{tabular}{|c|c|c|c|c|c|c|c|}
\hline & \begin{tabular}{|c|}
$\begin{array}{c}\text { Durée totale } \\
\text { (s) }\end{array}$ \\
\end{tabular} & $\begin{array}{c}\text { Durée utilisée } \\
\text { (s) }\end{array}$ & $\mathrm{N}^{\text {bre }}$ de SAs & $\mathrm{N}^{\text {bre }}$ de syll. & Syll./SA & $\mathbf{s} / \mathbf{S A}$ & \begin{tabular}{|c|}
$\begin{array}{c}\text { Débit } \\
\text { (syll./s) }\end{array}$ \\
\end{tabular} \\
\hline Rémie & 199,9 & 199,9 & 195 & 632 & 3,19 & 0,61 & 5,19 \\
\hline Éliane & \multirow{3}{*}{882,4} & 71,5 & 101 & 315 & 3,59 & 0,72 & 4,67 \\
\hline Patrice & & 23,03 & 35 & 123 & 3,52 & 0,79 & 5,04 \\
\hline Roland & & 129,19 & 167 & 547 & 3,52 & 0,79 & 4,24 \\
\hline Lucie & \multirow{2}{*}{323,3} & 64,68 & 101 & 305 & 3,03 & 0,64 & 4,73 \\
\hline Raymond & & 59,31 & 95 & 229 & 2,47 & 0,62 & 3,96 \\
\hline MOY. & & 91,27 & 115,67 & 358,5 & 3,22 & 0,69 & 4,64 \\
\hline
\end{tabular}

La durée utilisée des enregistrements québécois varie de 56,65 s (Charles) à 143,5s (Mireille), avec une moyenne de 94,84 s par personne (Tableau 4). Le nombre de SAs analysés varie de 107 (Gabrielle) à 286 (Mireille), et la moyenne constitue 164 groupes accentuels. Les nombres minimal et maximal de syllabes analysées appartiennent aux mêmes locutrices : 285 syll. et 828 syll. respectivement. La moyenne du nombre de syllabes analysées dans le sous-corpus québécois est de 534 syll. La longueur moyenne des SAs est de 3,28 syll. Le plus grand nombre de syllabes dans un SA a été observé chez Jules (3,85 syll.), et le nombre le plus petit, chez Gabrielle (2,66 syll.). Cependant, c'est Caroline qui a énoncé les SAs les plus longs $(0,7 \mathrm{~s})$, et Mireille qui a produit les groupes les plus courts $(0,5 \mathrm{~s})$. La durée moyenne des SAs est de 0,59 s dans le sous-corpus québécois. Le débit le plus rapide a été observé chez Charles (6,43 syll./s), tandis que le débit le plus lent, chez Gabrielle (4,56 syll./s), avec une moyenne de 5,63 syll./s pour l'ensemble du sous-corpus FQ.

Tableau 4. Découpage, longueur et durée des SAs : corpus FQ.

\begin{tabular}{|c|c|c|c|c|c|c|c|}
\hline & $\begin{array}{c}\text { Durée totale } \\
(\mathbf{s})\end{array}$ & $\begin{array}{c}\text { Durée utilisée } \\
(\mathbf{s})\end{array}$ & $\mathbf{N}^{\text {bre }}$ de SAs & $\mathbf{N}^{\text {bre }}$ de syll. & Syll./SA & s/SA & $\begin{array}{c}\text { Débit } \\
\text { (syll./s) }\end{array}$ \\
\hline Mireille & 225,8 & 143,5 & 286 & 828 & 2,90 & 0,50 & 5,77 \\
\hline Caroline & 191,37 & 124,67 & 178 & 664 & 3,73 & 0,70 & 5,33 \\
\hline Charles & 105,21 & 56,65 & 111 & 364 & 3,28 & 0,51 & 6,43 \\
\hline Gabrielle & 102,9 & 62,5 & 107 & 285 & 2,66 & 0,58 & 4,56 \\
\hline Jules & 216 & 86,6 & 133 & 512 & 3,85 & 0,65 & 5,91 \\
\hline Philippe & 333,9 & 95,13 & 169 & 551 & 3,26 & 0,56 & 5,79 \\
\hline MOY. & & $\mathbf{9 4 , 8 4}$ & $\mathbf{1 6 4}$ & $\mathbf{5 3 4}$ & $\mathbf{3 , 2 8}$ & $\mathbf{0 , 5 9}$ & $\mathbf{5 , 6 3}$ \\
\hline
\end{tabular}

En comparant les deux sous-corpus, on note qu'au Québec, la longueur des SAs est légèrement plus grande (3,28 syll./SA) qu'en Ontario ( 3,22 syll./SA), ce qui n'est pas une différence significative (test $t: \mathrm{t}=-0,234 ; \mathrm{p}=0,820)$. Cependant, la durée des SAs démontre le rapport inverse ( $0,59 \mathrm{~s}$ en FQ c. 0,69 en FW); cette différence a été confirmée statistiquement (test $t: \mathrm{t}=2,39 ; \mathrm{p}=0,038$ ). Les différences de longueurs et de durées des $\mathrm{SAs}$ suggèrent un débit plus rapide dans le sous-corpus québécois $(5,56 \mathrm{syll} . / \mathrm{s})$ que dans le sous-corpus ontarien $(4,64 \mathrm{syll} . / \mathrm{s})$, ce qui a été confirmé statistiquement (test $t: \mathrm{t}=-3,094 ; \mathrm{p}=0,011$ ).

\subsection{Typologie syllabique}

L'analyse des structures syllabiques montre qu'elles sont identiques dans les variétés étudiées (Tableau 5). Leur distribution est presque analogue, avec la syllabe du type CV qui domine : 56,95\% en Ontario et 57,33\% au Québec. La structure syllabique suivante la plus fréquente est CVC : 16,69\% en Ontario et 14,13\% au Québec. Suivent la structure V en FW (10,65\%) et CC(C)V en FQ (12,92\%), et V en FQ $(9,15 \%)$ et CC(C)V en FW (10,37 \%). Le taux 
de syllabes du type $\mathrm{CC}(\mathrm{C}) \mathrm{VC}$ équivaut à 3,93 \% selon nos données, et les proportions des autres syllabes fermées sont négligeables.

Tableau 5. Structures syllabiques observées dans les deux sous-corpus.

\begin{tabular}{|c|c|c|c|c|}
\hline \multirow{2}{*}{ Syllabe } & \multicolumn{2}{|c|}{ Ontario } & \multicolumn{2}{c|}{ Québec } \\
\cline { 2 - 5 } & $\mathbf{N}^{\text {bre }}$ & $\mathbf{\%}$ & $\mathbf{N}^{\text {bre }}$ & $\mathbf{\%}$ \\
\hline VC & 27 & 1,26 & 47 & 1,49 \\
\hline VC(C) & 1 & 0,05 & 1 & 0,03 \\
\hline $\mathbf{V}$ & 229 & 10,65 & 289 & 9,15 \\
\hline $\mathbf{C V C}$ & 359 & 16,69 & 446 & 14,13 \\
\hline $\mathbf{C V C C}(\mathbf{C})$ & 29 & 1,35 & 32 & 1,01 \\
\hline $\mathbf{C V}$ & 1225 & 56,95 & 1810 & 57,33 \\
\hline $\mathbf{C C}(\mathbf{C}) \mathbf{V C}$ & 61 & 2,84 & 124 & 3,93 \\
\hline $\mathbf{C C}(\mathbf{C}) \mathbf{V C C}(\mathbf{C})$ & 2 & 0,09 & 0 & 0,00 \\
\hline $\mathbf{C C}(\mathbf{C}) \mathbf{V}$ & 223 & 10,37 & 408 & 12,92 \\
\hline Total & 2151 & 100 & 3157 & 100 \\
\hline
\end{tabular}

\subsection{Distribution de durée syllabique et d'intensité vocalique}

\subsubsection{Proportions de durées des syllabes inaccentuées par rapport à la syllabe accentuée}

Les structures syllabiques étant identiques et le nombre de syllabes dans un SA étant très proche (3,28 syll. au Québec et 3,22 syll. en Ontario), il convient d'explorer la distribution des proportions des durées des syllabes. Pour pouvoir comparer nos résultats avec ceux des analyses antérieures, nous nous concentrons sur les SAs de quatre syllabes. Les résultats se basent sur 159 SAs en FQ et sur 105 SAs en FW et apparaissent dans la Fig. 1 à côté des résultats antérieurs de Robinson (1968) pour le français standard et le français ontarien en situation minoritaire. Ainsi, en comparant d'abord FQ et FW (Fig. 1), on note la similarité des proportions des durées des syllabes pénultièmes et antépénultièmes dans les deux variétés et la durée plus courte de la syllabe initiale en FW par rapport au FQ. Dans les deux sous-corpus, la durée de chaque syllabe suivante augmente, ce qui crée un pattern croissant. Les tests statistiques ont confirmé que les valeurs de durées ne sont pas différentes entre les sous-corpus (test $t: \mathrm{t} \geq-1,166 ; \mathrm{p} \geq 0,274$ ).

En comparant les nouveaux résultats ontariens avec ceux de Robinson (1968) (il s'agit des colonnes «Ontario » dans Fig. 1), il faut remarquer les proportions plus grandes des syllabes inaccentuées en 1968; le pattern de distribution des durées est aussi légèrement différent, les syllabes numéro deux et trois formant un plateau. Dans tous les corpus canadiens comparés, les proportions des durées des syllabes inaccentuées sont considérablement plus grandes par rapport au français standard. Ainsi est soutenue l'hypothèse selon laquelle les résultats des analyses du rythme prosodique en français canadien suggèrent une isochronie très syllabique à cause des proportions plus grandes des syllabes inaccentuées en comparaison du français standard.

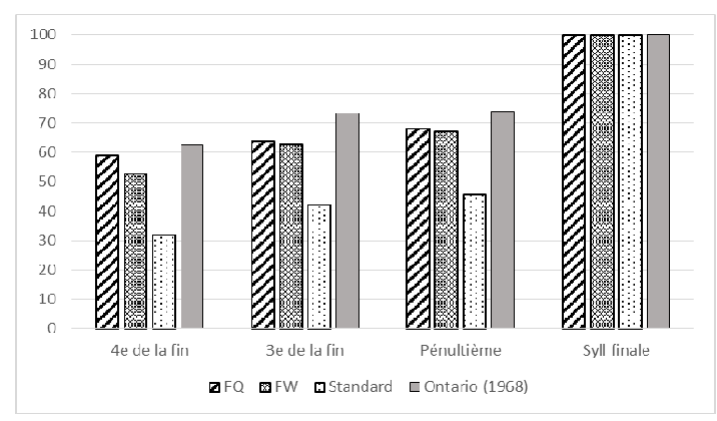

Fig. 1. Proportions des durées syllabiques par rapport à la syllabe finale dans quatre corpus.

\subsubsection{Distribution des durées syllabiques dans le syntagme accentuel}

Pour comparer les durées des syllabes accentuées, nous avons recalculé les durées syllabiques par rapport à la durée du SA. Les résultats apparaissent dans le Tableau 6, où on constate que la durée des syllabes finales en français canadien varie de $32,3 \%$ à 34,6 \%, alors qu'en français standard elle est de 45,5\%. Par conséquent, les proportions des syllabes inaccentuées sont plus grandes dans les variétés canadiennes (de 17,9\% à 23,8\%) qu'en français standard, où elles vont de $14,5 \%$ à 20,8 \%. Donc, si dans les variétés canadiennes et européenne le pattern duratif est croissant, les durées sont 
réparties de façon un peu plus égale dans les variétés canadiennes, ce qui crée un rythme plus égal et explique l'origine des résultats dans Kaminskaïa (2015).

Tableau 6. Proportion des durées syllabiques (\%) par rapport à la durée du SA.

\begin{tabular}{|l|c|c|c|c|}
\hline & $\mathbf{4}^{\mathbf{e}}$ de la fin & $\mathbf{3}^{\mathbf{e}}$ de la fin & Pénultième & Syllabe finale \\
\hline FQ & 20,4 & 22,1 & 23,5 & 34,6 \\
\hline FW & 17,9 & 21,3 & 22,8 & 34,0 \\
\hline $\begin{array}{l}\text { Français ontarien } \\
\text { (basé sur Robinson 1968: 166) }\end{array}$ & 20,2 & 23,7 & 23,8 & 32,3 \\
\hline $\begin{array}{l}\text { Français standard } \\
\text { (basé sur Robinson 1968 : 166) }\end{array}$ & 14,5 & 19,2 & 20,8 & 45,5 \\
\hline
\end{tabular}

\subsubsection{Intensité vocalique à travers le syntagme accentuel}

La réduction de la durée de la syllabe finale peut signaler un changement dans la nature de l'accent (Krivokapić 2013). Pour explorer la possibilité de convergence de cet aspect du rythme avec l'anglais, nous explorons la distribution de l'intensité dans les mêmes SA de quatre syllabes. La distribution de l'intensité vocalique normalisée est présentée dans la Fig. 2, où ressortent deux patterns très différents. En FQ, l'intensité des voyelles finale et pénultième est la plus basse, avec la voyelle numéro deux ayant l'intensité la plus grande. En FW, c'est l'intensité de la voyelle finale qui est la plus grande, la voyelle numéro deux étant la suivante selon la force. L'intensité la plus basse dans le sous-corpus ontarien est observée sur la voyelle initiale. Dans l'ensemble, à travers le SA, l'intensité augmente en FW et diminue en FQ. Ces différences ont été confirmées pour les syllabes finales du SA (test $t: \mathrm{t}=2,717 ; \mathrm{p}=0,009)$, mais non pas pour les syllabes non finales (test $t: \mathrm{t} \leq 0,273 ; \mathrm{p} \geq 0,092$ ).

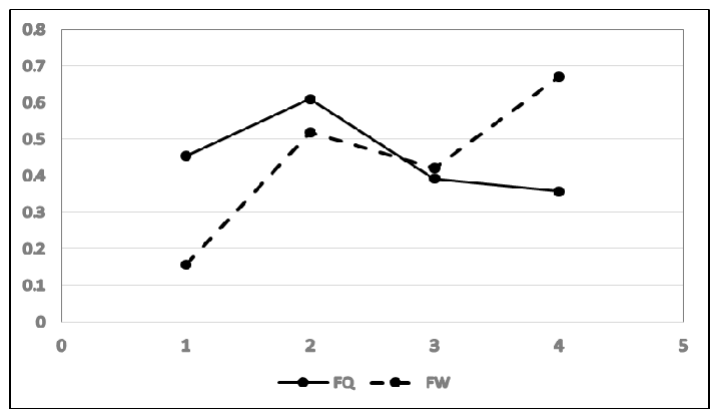

Fig. 2. Distribution de l'intensité vocalique normalisée à travers un groupe de quatre syllabes dans les deux sous-corpus. L'axe vertical représente les valeurs de cotes $Z$. Les chiffres « 1, 2, 3, 4 » renvoient aux syllabes dans un SA.

La hausse de l'intensité sur la deuxième voyelle dans nos données fait penser à l'accentuation secondaire (voir Di Cristo 1999, 2000, entre autres). L'accent rythmique secondaire, ou accent initial, est dit de nature mélodique en français standard (Jun et Fougeron 2002). Cependant, nos résultats suggèrent que l'accent initial en français canadien serait aussi marqué par l'intensité. Pour explorer cette hypothèse, nous avons identifié, à l'intérieur de chaque souscorpus, les SAs avec un seul accent final et les SAs avec un accent final et un accent rythmique secondaire sur la deuxième syllabe. Pour ce faire, nous nous sommes guidée par des critères auditifs, acoustiques et morphosyntaxiques (pour plus de détails, voir Jun et Fougeron 2002, Kaminskaïa 2005). Nous avons retenu seulement les SAs de continuité avec un contour mélodique montant pour réduire l'effet de la position du SA dans l'énoncé. En FW, nous avons analysé 18 SAs avec un accent final et 11 SAs avec deux accents. En FQ, le nombre de SAs avec un accent final est aussi de 18, alors que le nombre de syntagmes avec deux accents est de 16. Du point de vue de la structure syllabique, les données analysées sont très comparables.

Dans le sous-corpus ontarien, la différence entre les deux types de syntagmes est très nette (Fig. 3). Même si, dans les deux cas, la voyelle finale démontre une augmentation de l'intensité, dans les SAs avec un seul accent (ligne pointillée), les autres voyelles démontrent une intensité plus faible, et on observe un pattern croissant. Dans les SAs avec un accent secondaire, le pattern est montant-descendant-montant, et l'accent secondaire atteint un niveau d'intensité même plus grand que l'accent final. 


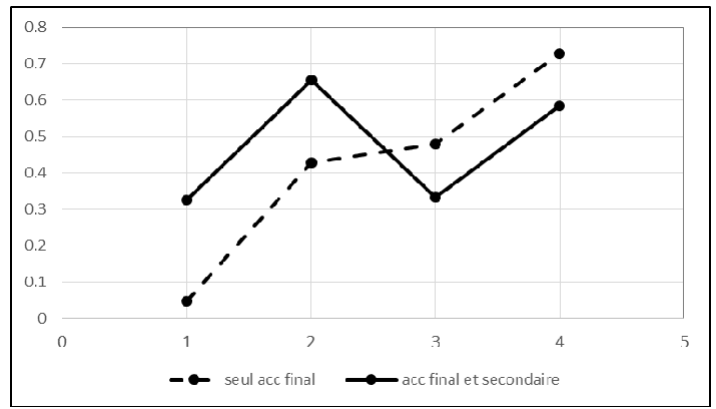

Fig. 3. Distribution de l'intensité normalisée dans les SAs avec un accent et avec deux accents, corpus Windsor. L'axe vertical représente les valeurs de cotes $Z$. Les chiffres « $1,2,3,4 »$ renvoient aux syllabes dans un SA.

L'image observée en FQ est très différente (Fig. 4) : le pattern d'intensité montant-descendant-montant caractérise les SAs à un seul accent (ligne pointillée), alors que les SAs à deux accents démontrent un pattern montant-descendant, avec la voyelle portant l'accent secondaire démontrant l'intensité maximale. Dans les deux cas, la valeur de l'intensité de la deuxième voyelle est identique.

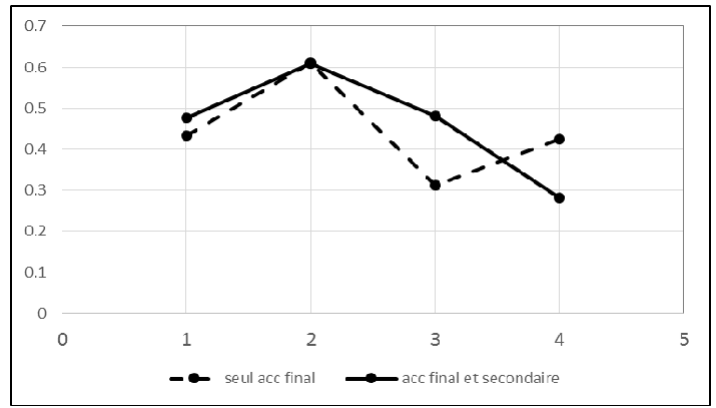

Fig. 4. Distribution de l'intensité normalisée dans les SAs avec un accent et avec deux accents, corpus Québec. L'axe vertical représente les valeurs de cotes $Z$. Les chiffres « 1, 2, 3, 4 » renvoient aux syllabes dans un SA.

Les tests ANOVA 2x2 ont vérifié l'effet des variables variété et nombre d'accents sur la variation de l'intensité. Pour la syllabe finale, l'effet de la variété est ressorti significatif $(F=6,630 ; p=0,013)$. Pour les syllabes non finales, nous n'avons pas observé d'importance des variables individuelles, ni d'interaction entre elles $(\mathrm{F} \leq 2,151 ; \mathrm{p} \geq 0,148)$.

\section{Conclusions et discussion}

Dans le contexte des études récentes portant sur le rythme prosodique en français québécois et en français ontarien en contact qui ont parfois suggéré des résultats contradictoires, mais surtout une rythmicité typiquement française dans les deux variétés, nous avons proposé ici d'examiner les aspects rythmiques qui permettent d'examiner la variation rythmique dans ces deux variétés apparentées plus profondément. L'analyse présentée visait à explorer la structure syllabique, la longueur et la durée des groupes accentuels, les rapports des durées syllabiques, et l'intensité des voyelles.

Les résultats ont démontré des similarités et des différences importantes. Du côté des similarités, nous avons observé une typologie syllabique presque identique, et des rapports des durées syllabiques semblables entre les deux variétés canadiennes. Ici, les proportions des durées des syllabes inaccentuées sont plus grandes qu'en français standard, mais plus petites que dans l'étude de Robinson (1968). D’une part, cela suggère que la durée reste toujours un corrélat acoustique important de l'accent final en français minoritaire, et d'autre part, cela explique pourquoi les mesures rythmiques dans les corpus canadiens suggèrent une isochronie syllabique plus forte que dans les corpus standard. Du côté des différences, notons aussi que la durée des SAs et le débit (les deux étant reliés) varient entre les variétés de façon significative : les locuteurs franco-ontariens parlent plus lentement (voir aussi Kaminskaïa 2015) et leurs groupes rythmiques sont plus longs en termes de temps. Cependant, les SAs en FW sont plus courts en termes de nombre de syllabes.

L'analyse de l'intensité vocalique a fourni des résultats qui non seulement caractérisent différemment les souscorpus, mais font penser au changement de la nature de l'accentuation en français ontarien en contact intense avec l'anglais. Ainsi, en FW, l'intensité semble faire partie de la mise en relief des deux accents, final et initial : ici, la syllabe finale portant l'accent primaire de durée paraît dotée, en plus du rallongement, d'une intensité importante (résultat confirmé statistiquement), et la syllabe portant l'accent secondaire est également énoncée avec plus de force. 
Ces observations vont de pair avec celles de Cichocki et al. (2014) qui concluent que, dans la variété du français acadien en contact parlé au sud-est du Nouveau-Brunswick, «[the] significant role played by intensity suggests that intensity is an acoustic cue of prominence ». Si l'on prend en considération que la forme des contours intonatifs correspond aux patterns d'intensité en FW (un contour plat-montant pour les SAs avec un accent et un contour montant-descendant-montant pour les SAs avec deux accents (Jun et Fougeron 2002), on peut conclure que la mélodie (et l'intensité) sont les corrélats acoustiques de l'accent initial, et que la durée, la mélodie et l'intensité sont les corrélats acoustiques de l'accent primaire final en français ontarien.

Dans la variété québécoise, les patterns d'intensité observés sur les syntagmes avec un seul accent et avec deux accents sont plus curieux. La deuxième syllabe est la plus forte dans les deux types de SAs, mais la syllabe finale est la plus faible dans les SAs avec deux accents, et dans les SAs avec un seul accent, l'intensité monte légèrement à la fin. Ce que nous tirons de ces résultats est que l'intensité ne participe pas à l'accentuation finale en FQ. En ce qui concerne l'accent secondaire, vu que l'intensité augmente dans les deux types de SAs (avec un ou avec deux accents) et étant donné les contours mélodiques montants-descendants-montants qui accompagnent les SAs avec deux accents, on peut supposer que c'est tout de même la mélodie qui marque l'accent initial dans cette variété. Au regard de ces observations, les résultats québécois suivent la description traditionnelle du français européen, où les corrélats acoustiques de l'accent sont la durée et la mélodie (Cumming 2011; Di Cristo 1999, 2000; Hirst et Di Cristo 1997 entre autres). L'augmentation de l'intensité sur la deuxième syllabe en FQ devrait être explorée plus amplement.

Ainsi, les sous-corpus FQ et FW semblent se distinguer selon la nature de l'accent primaire : à la durée et à la mélodie le FW ajoute l'intensité. L'intensité semble également participer dans la mise en relief de l'accent initial dans cette variété. Une étude visant la perception des proéminences permettrait d'explorer l'apport de ces corrélats dans l'accentuation en français canadien. Une étude incluant tous les participants des sous-corpus est aussi nécessaire pour confirmer nos observations. L'effet des variables d'âge et de sexe sur la nature des accents devrait également être exploré, vu leur importance dans les études antérieures du rythme prosodique en français canadien.

\section{Notes}

1. La classification traditionnelle des langues selon le rythme oppose les langues à rythmicité/isochronie syllabique (par ex., le français et l'espagnol) aux langues au rythme accentuel (par ex., l'anglais et l'allemand) (Abercrombie 1967; Pike 1945).

2. Les méthodes utilisant les mesures rythmiques s'appuient sur l'hypothèse selon laquelle elles reflètent les propriétés phonologiques des langues à rythmicités différentes (Dauer 1987). Par exemple, les langues à isochronie syllabique présentent une régularité des intervalles syllabiques grâce à la préférence de la structure syllabique CV et à l'absence de réduction vocalique, entre autres. Par contre, les langues à rythmicité accentuelle présentent une régularité des intervalles accentuels, ce qui est possible grâce à la réduction des voyelles inaccentuées et à la variabilité des structures syllabiques. Ces caractéristiques se traduisent, pour les langues à rythmicité syllabique, en une variabilité plus petite entre les durées des intervalles vocaliques et consonantiques (les écarts-types plus petits, $\Delta \mathrm{V}$ et $\Delta \mathrm{C}$ ) et en une proportion plus grande des intervalles vocaliques (\%V) (Ramus et al. 1999). C'est l'inverse pour les langues à isochronie accentuelle. À côté des $\Delta \mathrm{V}$ et $\Delta \mathrm{C}$, les mesures normalisant le débit de la parole ont été proposées : VarcoV (White et Mattys 2007) et VarcoC (Dellwo 2006). En plus, l'indice de variabilité des paires (PVI, Low et al. 2000) et ses variantes normalisées calculées à partir des intervalles vocaliques (nPVI-V) ou consonantiques (nPVI-C, Grabe et Low 2002) ont été largement adoptés par des chercheurs. Finalement, le modèle basé sur les PVI modifiés et prenant en considération à la fois les mesures vocaliques et les mesures consonantiques a été avancé par Bertinetto et Bertini $(2008,2010)$. Appelée Control and Compensation model, cette approche distingue les langues où le contrôle articulatoire est plus fort (le français) ou plus faible (l'anglais) et où les durées des intervalles sont donc maintenues (contrôlées) ou bien compensées à cause des réductions et des coarticulations. C'est le rapport entre les mesures vocaliques (CCI-Vs) et consonantiques (CCI-Cs) qui détermine la rythmicité : les valeurs proches indiquent un rythme syllabique, alors que les valeurs plus grandes des intervalles vocaliques par rapport aux intervalles consonantiques suggèrent un rythme accentuel. Nous renvoyons le lecteur aux sources originelles pour une discussion théorique, pour les informations sur les calculs et pour les détails méthodologiques.

Le plus important dans toutes ces méthodes proposées est la vision commune de la rythmicité comme continuum, au lieu de l'opposition entre les langues à rythmicité accentuelle et les langues au rythme syllabique.

3. Les analyses du rythme prosodique s'appuient sur les durées des intervalles vocaliques, et la durée de la voyelle est intégralement liée à la durée de la syllabe.

4. Les pauses contribuent à la variation rythmique, mais elles n'ont pas été prises en considération à cause d'une grande variabilité interindividuelle propre à la parole spontanée.

5. Les noms des participants sont modifiés pour garder leur anonymat.

Nous remercions les évaluateurs anonymes pour leurs commentaires précieux, et François Poiré pour l'accès aux données. Cette étude a été subventionnée par Lois Claxton Humanities and Social Sciences Award (Université de Waterloo 2014-2016).

\section{Références bibliographiques}

Abercrombie, D. (1967). Elements of General Phonetics. Edinburgh: Edinburgh University Press.

Bertinetto, P. M. et Ch. Bertini. (2008). On modeling the rhythm of natural languages. Dans Barbosa, P. A., Madureira, S. et C. Reis (dir.) Proceedings of the 4th International Conference on Speech Prosody (May 6-9, 2008), Campinas, Brasil, p. 427-430.

Bertinetto, P. M. et Ch. Bertini. (2010). Towards a unified predictive model of natural language rhythm. Dans Russo, M. (dir.) Prosodic Universals. Comparative studies in rhythmic modeling and rhythm typology. Napoli: Aracne, p. 43-77. 
Boersma, P. et D. Weenink. (2016). PRAAT: Doing phonetics by computer. Accédé de www.fon.hum.uva.nl/praat

Boudreault, M. (1968). Rythme et mélodie de la phrase parlée en France et au Québec. Québec: Presses de l’Université Laval.

Cichocki, W. (1997). Observations préliminaires sur le rythme en français acadien. Dans Dubois, L. et A. Boudreau (dir.) Les Acadiens et leur(s) langue(s) : quand le français est minoritaire. Moncton : Édition de l'Acadie, p. 63-73.

Cichocki, W. et D. Lepetit. (1986). Intonational variability in language contact. F0 declination in Ontarian French. Dans Sankoff, D. (dir.) Diversity and Diachrony. Amsterdam: John Benjamins, p. 239-247.

Cichocki, W., Selouani, S.-A. et Y. Perreault. (2014). Measuring rhythm in dialects of New Brunswick French: is there a role for intensity? Canadian Acoustics 42:3, 90-91.

Cumming, R. E. (2011). Perceptually informed quantification of speech rhythm in pairwise variability indices. Phonetica 68, 256277.

Dauer, R. M. (1987). Phonetic and phonological components of language rhythm. Actes du 11e International Congress of Phonetic Sciences (Estonia, Tallinn), 447-450.

Dellwo, V. (2006). Rhythm and speech rate: A variation coefficient for delta. Dans Karnowski, P. et I. Szigeti (dir.) Language and Language-Processing: Proceedings of the $38^{\text {th }}$ Linguistic Colloquium, p. 231-241.

Di Cristo, A. (1999). Vers une modélisation de l'accentuation du français (première partie). Journal of French Language Studies $9: 2,143-179$

Di Cristo, A. (2000). Vers une modélisation de l'accentuation du français (seconde partie). Journal of French Language Studies $10: 1,27-45$.

Durand, J., Laks, B et Ch. Lyche. (2009). Le projet PFC : une source de données primaires structurées. Dans Durand, J., Laks, B et Ch. Lyche (dir.) Phonologie, variation et accents du français. Paris : Hermès, p. 19-61.

Goldman, J.-Ph. (2011). EasyAlign: a friendly automatic phonetic alignment tool under Praat. Actes de la $12^{\text {th }}$ Annual Conference of the International Speech Communication Association (INTERSPEECH 2011), Florence, Italy, p. 3233-3236.

Grabe, E. et E. L. Low. (2002). Durational variability in speech and the rhythm class hypothesis. Dans Warner, N. et C. Gussenhoven (dir.) Papers in Laboratory Phonology 7, 515-546.

Hirst, D. et A. Di Cristo (1997). L'accentuation non-emphatique en français : stratégies et paramètres. Dans Perrot, J. (dir.) Polyphonie pour Iván Fónagy. Paris-Montréal : L'Harmattan, p. 71-101.

Jun, S.-A. et C. Fougeron. (2002). Realizations of accentual phrase in French intonation. Probus 14, 147-172.

Kaminskaïa, S. (2005). Une étude comparée de l'intonation de la parole spontanée dans deux dialectes du français sur deux niveaux prosodiques. Thèse de doctorat, Université Western Ontario.

Kaminskaïa, S. (2012). Le timing des pics mélodiques en français spontané en milieu minoritaire. Dans Caxaj-Ruiz, P. (dir.), Proceedings of the 2012 Canadian Linguistic Association Annual Conference, http://homes.chass.utoronto.ca/ claacl/actes2012/Kaminskaia 2012.pdf.

Kaminskaïa, S. (2013). Intonation of Ontario French in a minority setting: a study comparing two age groups. The French Review $87: 2,107-127$.

Kaminskaïa, S. (2014). Quantification of speech rhythm in Canadian French in a minority setting. The Italian Journal of Linguistics/Rivista di Linguistica 26:1, 93-133.

Kaminskaïa, S. (2015). L'apport du débit à l'étude du rythme phonétique à l'aide des mesures rythmiques : une étude de deux variétés du français laurentien. Faits de langues 45, 161-185.

Kaminskaïa, S. et F. Poiré. (2012). Prosodie du français laurentien en milieu minoritaire: le corpus Windsor. Dans Simon, A. C. (dir.) La variation prosodique régionale en français, Chapitre 8. Bruxelles : De Boeck, coll. "Champs linguistiques", p. 159-178.

Kaminskaïa, S., Tennant, J. et A. Russell. (2009). Variation rythmique en français québécois et ontarien. Communication faite au congrès annuel de Atlantic Provinces Linguistics Association, Universite Memorial, St. John's, Terre Neuve, Canada, novembre 2009.

Kaminskaïa, S., Tennant, J. et A. Russell. (2015). Prosodic rhythm in Ontario French. Journal of French Language studies 26:2, 183-208.

Krivokapić, J. (2013). Rhythm and convergence between speakers of American and Indian English. Dans Grice, M., et D. Mücke (dir.) Laboratory Phonology 4/1: Rhythm, speech timing, and perceptual processing, 39-66.

Lieberman, Ph. (1960). Some Acoustic Correlates of Word Stress in American English. The Journal of the Acoustical Society of America 32:4, 451-454.

Low, E. L., Grabe, E. et F. Nolan. (2000). Quantitative characterizations of speech rhythm: Syllable-timing in Singapore English. Language and Speech $43: 4,377-401$.

Mzemba, D. (2016). L'intonation et contact de langues: le cas de l'intonation du français parlé à Rivière-la-Paix, Alberta, Thèse de doctorat, Université Western.

Obin, N., Avanzi, M., Bordal, G. et A. Bardiaux. (2012). Regional Variations of Speech Rhythm in French. In Search of Lost Times. Dans Ma, Q., Ding, H. et D. Hirst (dir.) Proceedings of Speech Prosody 6. http://www.speechprosody2012.org/uploadfiles/file/sp2012 submission 224.pdf

Poiré, F. et S. Kelly. (2003). Présentation de l'étude du français, langue minoritaire, dans le sud-ouest ontarien dans le cadre du PFC. Communication faite à Phonologie et phonétique du français: données et théorie, Paris, Maison des sciences de l'homme, décembre 2003.

Ramus, F., Nespor, M. et J. Mehler. (1999). Correlates of linguistic rhythm in the speech signal. Cognition 73, $265-292$.

Robinson, L. (1968). Étude du rythme syllabique en français canadien et en français standard. Dans Léon, P. (dir.) Recherches sur la structure phonique du français canadien (Studia Phonetica 1). Paris : Didier, p. 161-174.

Schwab, S. et I. Racine. (2013). Le débit lent des Suisses romands : mythe ou réalité? Journal of French Language Studies 23, 281295.

Tennant, J. (2011). Rythme prosodique et contact des langues dans le français ontarien. Dans Martineau, F. et T. Nadasdi (dir.) Le français en contact. Hommages à Raymond Mougeon. Québec : Presses de l’Université Laval, p. 355-373.

Vinay, J.-P. (1955). Aperçu des études de phonétique canadienne. Dans Société du parler français au Canada, Études sur le parler français au Canada. Québec : Presses de l'Université Laval, p. 61-82.

White, L. et S. L. Mattys. (2007). Calibrating rhythm: First and second language studies. Journal of Phonetics 35, 501-522.

Williams, D. et F. Poiré. (2007). Predicting Vowel Duration in Spontaneous Canadian French Speech. Actes de INTERSPEECH 2007, Anvers, Belgique, p. 1018-1021. 